\title{
Exploring the differences between early-onset gastric cancer and traditional-onset gastric cancer
}

\author{
Anwar Giryes ${ }^{1}$, Hani Oweira ${ }^{2}$, Meinrad Mannhart ${ }^{3}$, Michael Decker ${ }^{4}$, Omar Abdel-Rahman $^{5,6}$ \\ ${ }^{1}$ Swiss Medical Clinic, Zürich, Switzerland; ${ }^{2}$ Gastrointestinal Tumor Zentrum, Zürich, Switzerland; ${ }^{3}$ Department of Oncology, Center of Zug, Zug, \\ Switzerland; ${ }^{4}$ Oncology Department, Zentrum für Integrative Onkologie, Zürich, Switzerland; ${ }^{5}$ Clinical Oncology Department, Faculty of Medicine, \\ Ain Shams University, Cairo, Egypt; 'Department of Oncology, Tom Baker Cancer Centre, University of Calgary, Calgary, Alberta, Canada \\ Contributions: (I) Conception and design: O Abdel-Rahman; (II) Administrative support: All authors; (III) Provision of study material or patients: All \\ authors; (IV) Collection and assembly of data: O Abdel-Rahman; (V) Data analysis and interpretation: O Abdel-Rahman; (VI) Manuscript writing: \\ All authors; (VII) Final approval of manuscript: All authors. \\ Correspondence to: Dr. Omar Abdel-Rahman, MD. Department of Oncology, University of Calgary, Tom Baker Cancer Centre, Calgary, Alberta, \\ Canada. Email: omar.abdelrhman@med.asu.edu.eg; omar.abdelsalam@ahs.ca.
}

Background: The current study sought to explore the potential clinical, epidemiological and genetic differences between early-onset gastric cancer (E-gastric cancer: defined as 20-39 years) and traditionalonset gastric cancer ( $T$-gastric cancer: defined as $\geq 40$ years).

Methods: Datasets from the following sources were searched: Surveillance, Epidemiology and End Results database [2000-2014], Behavioral Risk Factor Surveillance Survey and the cancer genome atlas (TCGA). Clinicopathological characteristics, trends, and genetic findings were compared between E-gastric cancer and T-gastric cancer. Moreover, correlations with relevant risk factors were sought after.

Results: A total of 95,323 gastric cancer patients were identified in the period from 2000 to 2014. While T-gastric cancer was decreasing during the study period $(-1.4 ; \mathrm{P}<0.05)$, E-gastric cancer was stable during the study period. E-gastric cancer is less prevalent in males $(51.1 \%$ vs. $61.0 \% ; \mathrm{P}<0.0001)$, and white patients (68.9\% vs. $71.4 \% ; \mathrm{P}<0.0001)$. E-gastric cancer patients usually present with poorly differentiated histology (55.3\% vs. $48.0 \% ; \mathrm{P}<0.0001)$ as well as more aggressive histological subtypes (e.g., diffuse histology or linitis plastica). No difference can be detected with regards to risk factor correlations between E-gastric cancer and T-gastric cancer. Only four patients with E-gastric cancer were available in the provisional TCGA dataset at the time of the study.

Conclusions: E-gastric cancer is a potentially distinct disease entity with specific clinicopathological and trend patterns compared to conventional T-gastric cancer. Further studies are needed to explore the potential etiologic basis as well as to investigate the clinical consequences of this distinction. The impact of this distinction on minority populations requires further assessment as well.

Keywords: Gastric cancer; early-onset; clinical criteria; biological criteria

Submitted May 21, 2018. Accepted for publication Jun 27, 2018.

doi: 10.21037/jgo.2018.06.11

View this article at: http://dx.doi.org/10.21037/jgo.2018.06.11

\section{Introduction}

In spite of the important advances in gastric cancer diagnosis and treatment in the past decades, it remains an important global cause of mortality and morbidity (1). It still ranks as the $5^{\text {th }}$ most common malignancy in the world; and according to GLOBOCAN, about 1 million new cases of gastric cancer were diagnosed in 2012 (2). Moreover, it the $3^{\text {rd }}$ largest cause of cancer mortality with the highest mortality rates reported in eastern Asian countries (3).

Gastric adenocarcinoma cases were stratified based on multiple factors including location (cardia $v s$. noncardia), histology as well as biology $(4,5)$. Despite the 
increasing recognition that gastric cancer arising in the young has peculiar characteristics, the clinicopathological, epidemiological and genetic characteristics of early-onset gastric cancer (E-gastric cancer) were not thoroughly evaluated before (6).

The Surveillance, Epidemiology and End Results (SEER) database was explored to evaluate the pathological and clinical characteristics of E-gastric cancer (defined in this study as patients with age at diagnosis from 20-39 years) and traditional-onset (defined in this study as patients with age at diagnosis $\geq 40$ years) gastric cancer (T-gastric cancer). The cutoff of 40 years was used because previous reports used this cutoff $(6,7)$. Subsequently, the rates of age-adjusted gastric cancer were compared with the rates of some of the potential risk factors for gastric cancer available from the Behavioral Risk Factor Surveillance Survey (BRFSS). In line with the above analyses, the molecular structures of E-gastric cancer and T-gastric cancer were evaluated through The Cancer Genome Atlas (TCGA) to further clarify the potential differences between these two entities.

\section{Methods}

\section{SEER data analysis}

The most recent submission of the SEER database contains data from 18 registries (8); this submission was accessed through SEER*stat V. 8.3.4. Through "case listing session" in SEER*stat, baseline clinicopathological characteristics of all gastric cancer cases diagnosed 2000-2014 were reviewed. Cases were classified into two cohorts: earlyonset disease (20-39 years) and traditional-onset disease ( $\geq 40$ years). Through Chi-square testing, baseline characteristics were compared between the two cohorts. The following information was collected for each patient: ethnicity, subsite (cardia versus non-cardia), SEER summary stage (localized, regional, distant or unknown), histology, grade, and gender. All statistical analyses were conducted through SPSS v. 20 (IBM, NY, USA); P values $<0.05$ represented statistical significance.

Through the "rate session" in SEER*stat, age-adjusted incidence rates per 100,000 were calculated. These were stratified by the two disease categories (E-gastric cancer versus T-gastric cancer). Annual percent change (APC) was then calculated for each disease category and it was stratified by ethnic group, stage at presentation and by the location of the tumor.

\section{BRFSS analysis}

BRFSS is an annual cross-sectional population-based survey (incorporating approximately 500,000 individuals each year). Full details of this survey were previously described and validated (9). Through accessing BRFSS dataset, rates of potential gastric cancer risk factors (e.g., smoking, obesity or heavy alcohol consumption) were determined for each state in 2014. Assessment of the 2014 rates of E-gastric cancer and T-gastric cancer were conducted in parallel for the available state SEER registries. After confirmation of normality through Shapiro-Wilk test, Pearson correlation was conducted between risk factor rates and gastric cancer rates.

\section{TCGA analysis}

The most recent provisional TCGA gastric adenocarcinoma dataset was accessed through Genomics Data Commons (GDC) portal and cBioPortal (10). This was done in order to study potential genetic differences between E-gastric cancer and T-gastric cancer. Both portals provide an easy access to the TCGA data with the possibility to produce visualization, analysis and possibly download of TCGA data in addition to other large-scale multidimensional genomic datasets (11). It allows exploration of clinical data, mutated genes as well as copy number alterations (CNAs).

\section{Results}

\section{Clinicopathological characteristics}

A total of 95,323 gastric cancer patients older than 20 years diagnosed 2000-2014 were found eligible and were included in the analysis. Of this cohort, 3,247 patients (3.4\%) have E-gastric cancer; while 92,076 patients (96.6\%) have T-gastric cancer. Comparing E-gastric cancer to T-gastric cancer, E-gastric cancer is less likely to occur in whites $(68.9 \%$ vs. $71.4 \% ; \mathrm{P}<0.0001)$, less likely to occur in males $(51.1 \%$ vs. $61.0 \%$; $\mathrm{P}<0.0001)$, more likely to be poorly differentiated $(55.3 \%$ vs. $48.0 \%$; $\mathrm{P}<0.0001)$; and more likely to have distant metastases $(48.3 \%$ vs. $32.5 \% ; \mathrm{P}<0.0001$ ). More aggressive histologies (e.g., diffuse histology or linitis plastica) and more probability for lymph nodal metastases are also more common in E-gastric cancer compared to T-gastric cancer $(\mathrm{P}<0.0001$ for both) (Table 1). 
Table 1 Baseline clinicopathological characteristics of early- and traditional-onset gastric cancer; SEER database: 2000-2014

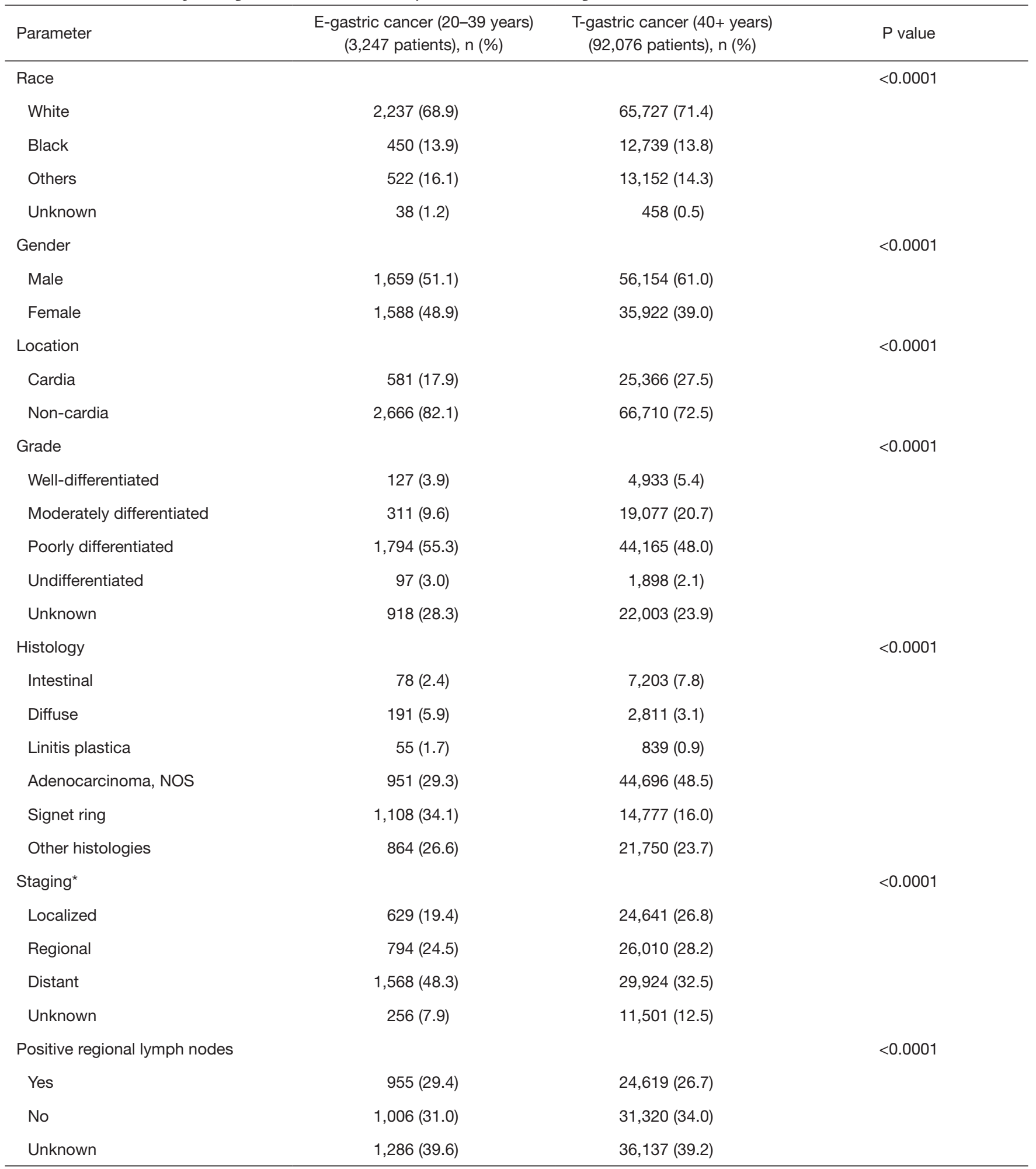

Table 1 (continued) 
Table 1 (continued)

\begin{tabular}{|c|c|c|c|}
\hline Parameter & $\begin{array}{c}\text { E-gastric cancer (20-39 years) } \\
(3,247 \text { patients), n (\%) }\end{array}$ & $\begin{array}{l}\text { T-gastric cancer (40+ years) } \\
\text { (92,076 patients), n (\%) }\end{array}$ & $P$ value \\
\hline Patients with distant metastases ${ }^{\star *}$ & & & Not applicable \\
\hline Brain & $10(0.3)$ & $196(0.2)$ & \\
\hline Surgery to the primary & & & 0.774 \\
\hline Yes & $1,351(41.6)$ & 38,605 (41.9) & \\
\hline No & $1,830(56.4)$ & $51,465(55.9)$ & \\
\hline No/unknown & $1,290(39.7)$ & $56,930(61.8)$ & \\
\hline Radiotherapy & & & $<0.0001$ \\
\hline Yes & $714(22.0)$ & $19,068(20.7)$ & \\
\hline No/unknown & $2,533(78.0)$ & $73,008(79.3)$ & \\
\hline
\end{tabular}

*, according to SEER summary staging system; **, among metastatic patients diagnosed after 2010. SEER, The Surveillance, Epidemiology and End Results.

\section{Trend analysis}

Across the period from 2000 to 2014, E-gastric cancer increased with an APC of 1.0 (non-significant P value). On the other hand, T-gastric cancer decreased in the same period at an APC of $-1.4(\mathrm{P}<0.05)$.

When stratifying the incidence trends of E-gastric cancer by SEER stages, localized and distant categories were increasing (at an APC of 3.2 and 1.5; non-significant $\mathrm{P}$ values); on the other hand, the regional category was declining at an APC of -1.0 (non-significant $\mathrm{P}$ value). Likewise, the incidence rates for T-gastric cancer stratified by the different stages were declining at APCs of -0.2 (localized), -3.3 (regional) and -0.3 (distant) (significant $\mathrm{P}$ value only for the regional stage) (Figure $1 A$ ).

When stratifying the incidence trends of E-gastric cancer by ethnicity, white race, black race and other races were declining at APCs of $-0.7,-1.2$ and -1.2 respectively (nonsignificant $\mathrm{P}$ value for the three categories). Likewise, the incidence rates for T-gastric cancer stratified by the different stages were declining at APCs of $-1.2,-2.0$ and $-3.1(\mathrm{P}<0.05$ for the three categories) (Figure 1B).

When stratifying the incidence trends of E-gastric cancer by location, both cardia and non-cardia locations were increasing at APCs of 1.3 and 0.9 respectively (nonsignificant $\mathrm{P}$ value for the two categories). Likewise, the incidence rates for T-gastric cancer stratified by the location was increasing for cardia location at an APC of 0.3 (nonsignificant $\mathrm{P}$ value) and declining at non-cardia location at an APC of $-2.0(\mathrm{P}<0.05)$ (Figure 1C).

\section{BRFSS analysis}

In order to elaborate further on the potential differences between E-gastric cancer and T-gastric cancer, an assessment of the correlation between some recognized risk factors (obesity, heavy drinking, and smoking) for gastric cancer and incidence of E-gastric cancer and T-gastric cancer were conducted. As illustrated in Table 2, there was a strong positive correlation for both E-gastric cancer and T-gastric cancer with heavy drinking $(\mathrm{P}=0.027$ and 0.008 respectively). On the other hand, there was no correlation 

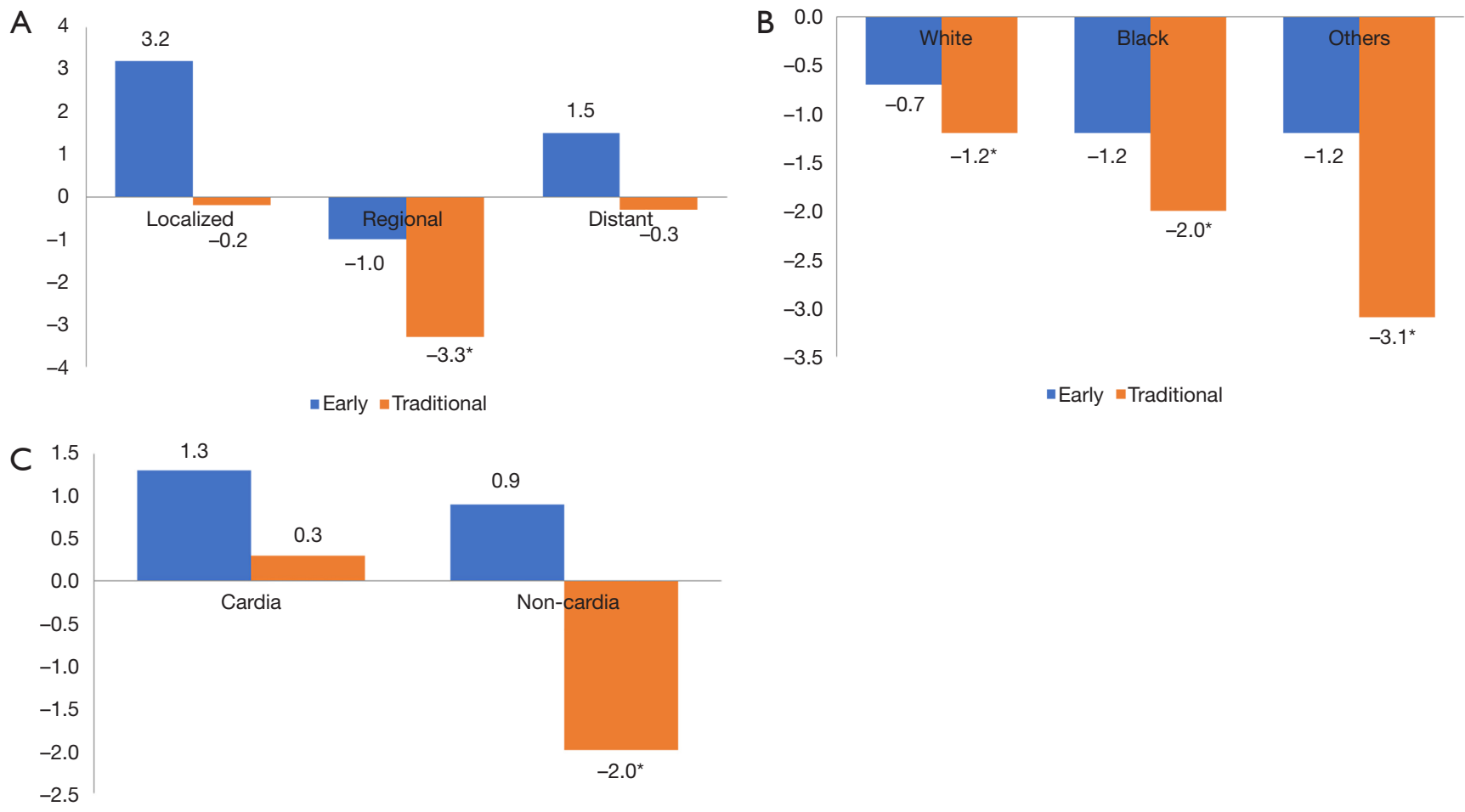

- Early $=$ Traditional

Figure 1 Trends in gastric cancer incidence. (A) By subtype (early vs. traditional) and SEER stage; (B) by subtype (early vs. traditional) and race; (C) by subtype (early vs. traditional) and location (Y-axis represents APC and * indicates significant APC). APC, annual percent change.

Table 2 Pearson correlation between age-adjusted rate of gastric cancer (stratified by early and traditional cases) and some of the possible risk factors of gastric cancer reported in BRFSS in 2014

\begin{tabular}{lcccc}
\hline \multirow{2}{*}{ Risk factors } & \multicolumn{2}{c}{ E-gastric cancer } & \multicolumn{2}{c}{ T-gastric cancer } \\
\cline { 2 - 5 } & $\mathrm{R}$ & $\mathrm{P}$ value & $\mathrm{R}$ & 0.221 \\
\hline Current smoking & 0.089 & 0.794 & 0.751 & 0.513 \\
Heavy alcohol drinking & 0.661 & 0.027 & 0.008 \\
Obesity** $^{*}$ & $<0.001$ & 1 & 0.051 & 0.882 \\
\hline
\end{tabular}

${ }^{*}$, defined as: adult men having more than 14 drinks per week and adult women having more than 7 drinks per week; ${ }^{* *}$, defined as: body mass index $\geq 30$. BRFSS, Behavioral Risk Factor Surveillance Survey; E-gastric cancer, early-onset gastric cancer; T-gastric cancer, traditional-onset gastric cancer.

between obesity or smoking and either E-gastric cancer or T-gastric cancer.

\section{TCGA analysis}

The clinical, pathological and trend differences between E-gastric cancer and T-gastric cancer suggested possible differences in genetic composition between the two disease entities. TCGA provisional dataset was explored through cBioPortal and GDC portal. There were 443 gastric adenocarcinoma cases included in this dataset. However, only four cases were $\leq 40$ years old. Because of the small number in the E-gastric cancer cohort, it is not feasible to conduct statistical analyses about differences in the pattern 
or frequency of mutated genes between both groups.

\section{Discussion}

The current study explored the potential peculiarities associated with gastric cancer developing at an early age compared with that developing at a later age. It showed that E-gastric cancer has different clinicopathological characteristics and trend patterns compared to T-gastric cancer. Moreover, genetic makeup seems to be different between both categories (although this cannot be proven statistically because of sample size considerations). On the other hand, and within the limitations of the current data and methodology, there was no evidence of a difference in the correlation with some established risk factors between both categories.

The current analysis suggested that E-gastric cancer is associated with unfavorable baseline clinicopathological features (more advanced stage and more aggressive histology compared to T-gastric cancer). This is consistent with previously published data about gastric cancer in the young and very young (12). Possible reasons for this difference might include different biology as well as a tendency to delayed diagnosis of gastric cancer in the young (because practitioners usually have a lower index of suspicion for gastric cancer diagnosis among younger patients compared to older patients) (13).

Likewise, trend analysis from the current study indicated that in spite of declining incidence trends among T-gastric cancer, overall incidence trends for E-gastric cancer were almost stable in the past 15 years. This might be explained by a yet unnoticed change in risk factor epidemiology (e.g., food patterns, or patterns of smoking) across the past 15 years which affected more the incidence of T-gastric cancer compare to E-gastric cancer.

Previous studies suggested that gastric cancer in the young and very young are associated more with hereditary tumor susceptibility syndromes $(14,15)$. Data from current TCGA analysis showed that both E-gastric cancer and T-gastric cancer harbor a considerable amount of genetic mutations as well as CNAs. This might indicate that gastric cancer arising in the young and very young might not be entirely explained out of hereditary etiologies.

Taken together, clinical, pathological and epidemiological analyses, as well as less association with white race, suggested that E-gastric cancer might be a phenotypically distinct entity from T-gastric cancer.

The limitations of this study are similar to those encountered with other studies based on administrative data. These include variations in disease coding and disease severity. However, the reporting of patient age is not expected to be affected by these variations. The limited number of E-gastric cancer in TCGA analysis also prevented proper statistical correlations. Moreover, the correlation between risk factors reported in BRFSS for each state and the respective incidence rates reported in SEER database for this state might be too crude to detect smaller differences. The absence of information about family history and hereditary cancer susceptibility is an additional limitation in the current study.

Further epidemiological and genetic studies focused on the potential differences between these two entities are needed. Likewise, further clinical studies are needed to explore the clinical consequences of dissecting E-gastric cancer as a distinct entity from the conventional T-gastric cancer.

In conclusion, E-gastric cancer is a potentially distinct disease entity with specific clinicopathological and trend patterns compared to conventional T-gastric cancer. The mechanisms underlying the differences between both disease entities are largely unknown. Further studies are needed to explore the potential etiologic basis as well as to investigate the clinical consequences of this distinction. The impact of this distinction on minority populations requires further assessment as well.

\section{Acknowledgements}

None.

\section{Footnote}

Conflicts of Interest: The authors have no conflicts of interest to declare.

\section{References}

1. Hamashima C. Current issues and future perspectives of gastric cancer screening. World J Gastroenterol 2014;20:13767-74.

2. Ferlay J, Soerjomataram I, Ervik M, et al. GLOBOCAN 2012 v1.0, Cancer Incidence and Mortality Worldwide: IARC CancerBase No. 11 [Internet]. Lyon, France: International Agency for Research on Cancer, 2013. (Accessed on 3/11/2017). Available online: http://globocan. iarc.fr 
3. Rahman R, Asombang AW, Ibdah JA. Characteristics of gastric cancer in Asia. World J Gastroenterol 2014;20:4483-90.

4. Galizia G, Lieto E, Auricchio A, et al. Comparison of the current AJCC-TNM numeric-based with a new anatomical location-based lymph node staging system for gastric cancer: A western experience. PLoS One 2017;12:e0173619.

5. Shim JH, Song KY, Jeon HM, et al. Is gastric cancer different in Korea and the United States? Impact of tumor location on prognosis. Ann Surg Oncol 2014;21:2332-9.

6. Strong VE, Russo A, Yoon SS, et al. Comparison of Young Patients with Gastric Cancer in the United States and China. Ann Surg Oncol 2017;24:3964-71.

7. Dhobi MA, Wani KA, Parray FQ, et al. Gastric cancer in young patients. Int J Surg Oncol 2013;2013:981654.

8. Surveillance, Epidemiology, and End Results (SEER) Program (www.seer.cancer.gov) SEER*Stat Database: Incidence - SEER 18 Regs Research Data + Hurricane Katrina Impacted Louisiana Cases, Nov 2016 Sub (19732014 varying) - Linked To County Attributes - Total U.S., 1969-2015 Counties, National Cancer Institute, DCCPS, Surveillance Research Program, Surveillance Systems Branch, released April 2017, based on the November 2016 submission.

Cite this article as: Giryes A, Oweira H, Mannhart M, Decker M, Abdel-Rahman O. Exploring the differences between early-onset gastric cancer and traditional-onset gastric cancer. J Gastrointest Oncol 2018;9(6):1157-1163. doi: 10.21037/ jgo.2018.06.11
9. Nelson DE, Holtzman D, Bolen J, et al. Reliability and validity of measures from the Behavioral Risk Factor Surveillance System (BRFSS). Soz Praventivmed 2001;46 Suppl 1:S3-42.

10. Grossman RL, Heath AP, Ferretti V, et al. Toward a Shared Vision for Cancer Genomic Data. N Engl J Med 2016;375:1109-12.

11. Cerami E, Gao J, Dogrusoz U, et al. The cBio Cancer Genomics Portal: An Open Platform for Exploring Multidimensional Cancer Genomics Data. Cancer Discovery 2012;2:401-4.

12. Merchant SJ, Kim J, Choi AH, et al. A rising trend in the incidence of advanced gastric cancer in young Hispanic men. Gastric Cancer 2017;20:226-34.

13. Isobe T, Hashimoto K, Kizaki J, et al. Characteristics and prognosis of gastric cancer in young patients. Oncol Rep 2013;30:43-9.

14. Slavin T, Neuhausen SL, Rybak C, et al. Genetic Gastric Cancer Susceptibility in the International Clinical Cancer Genomics Community Research Network. Cancer Genet 2017;216-217:111-9.

15. Hansford S, Kaurah P, Li-Chang H, et al. Hereditary Diffuse Gastric Cancer Syndrome: CDH1 Mutations and Beyond. JAMA Oncol 2015;1:23-32. 